\title{
TRAVELLING LATTICE WAVES IN A TOY MODEL OF LENNARD-JONES INTERACTION
}

\author{
BY \\ CHRISTINE R. VENNEY (Dept. of Mathematical Sciences, University of Bath, Bath, U. K.) \\ AND \\ JOHANNES ZIMMER (Dept. of Mathematical Sciences, University of Bath, Bath, U. K.)
}

\begin{abstract}
We consider an infinite lattice model, where particles interact with nearest neighbour (NN) and next-to-nearest neighbours (NNN); the NN and NNN springs act against each other to mimic the Lennard-Jones potential. The existence of subsonic waves homoclinic to exponentially small periodic oscillations is shown as well as the existence of supersonic periodic solutions. The proofs rely on methods from normal form and centre space analysis for the homoclinic solutions and centre manifold analysis for the periodic solutions.
\end{abstract}

1. Introduction. We analyse a one-dimensional chain of atoms, where both nearest neighbours (NN) and next-to-nearest neighbours (NNN) are linked by anharmonic springs,

$$
\begin{aligned}
c^{2} \ddot{x}(t)=U_{1}^{\prime}(x(t+1)-x(t))- & U_{1}^{\prime}(x(t)-x(t-1)) \\
& +g\left[U_{2}^{\prime}(x(t+2)-x(t))+U_{2}^{\prime}(x(t)-x(t-2))\right]
\end{aligned}
$$

(the notation is explained in Section 2). The configuration is such that the NN and NNN springs pull against each other, thus giving a poor man's version of the Lennard-Jones potential $a\left(-\left(\frac{b}{r}\right)^{6}+\left(\frac{b}{r}\right)^{12}\right)$, where $r$ is (in suitable units) the distance between two atoms.

Since in a physical situation, all atoms interact with each other, there is a clear physical motivation for including more than just NN interactions. We consider the simplest model by just including two kinds of springs but capture the attractive and repulsive behaviour of the Lennard-Jones potential by letting the springs act against each other with a ratio suggested by the linearised (harmonic) model. The analysis is confined to travelling waves.

This inclusion of NNN springs changes the nature of solutions drastically: a careful asymptotic analysis by Wattis [16] suggested that there are subsonic solitary waves with

Received February 25, 2012.

2010 Mathematics Subject Classification. Primary 37L10, 82C20. 
oscillatory decay. This is very different from the NN case, where solitary waves are supersonic and homoclinic to a constant state. We here give a rigorous proof of this conjecture, for a suitable range of parameters.

The core of the argument is a centre space analysis, in the framework developed by Iooss and Kirchgässner [7]. However, there are significant additional difficulties. A spectral investigation suggests a centre space analysis with the tools described in Lombardi's book [9]; this is since real eigenvalues are present which are absent in the pure NN problem of [5]. However, the tools of [9] are not directly applicable since (i) it is there assumed that the linearised operator is sectorial, which is not the case for the operator considered here (this is typical for lattice operators), and (ii) the centre space here is larger than for the problem discussed in [9].

We also give a proof, using centre manifold analysis, of the "complementary" result, namely, the existence of slightly supersonic periodic waves. Recently, such an existence result was proved differently by employing a linking argument [15. Again, this result is in contrast to the NN situation, where periodic waves are strictly confined to the subsonic regime.

Few other rigorous results seem to be available for lattice models with NNN interactions. We are only aware of one result for NNN interactions showing the existence of solutions homoclinic to exponentially small periodic waves, namely, the work by Calleja and Sire [1. They consider NN and NNN interaction for the Frenkel-Kontorova chain but with the two kinds of springs pulling in the same direction. The travelling wave equation for the Frenkel-Kontorova chain in suitable units is

$$
c^{2} \ddot{x}(t)=\gamma[x(t+1)-2 x(t)+x(t-1)+g(x(t+2)-2 x(t)+x(t-2))]-V^{\prime}(x(t)) .
$$

As far as the equation is concerned, the difference between (2) and the Fermi-Pasta-Ulam (FPU) chain studied here in the travelling wave formulation (1) is that the interaction between atoms is linear for the Frenkel-Kontorova (FK) chain but nonlinear for the FPU chain; the FK chain has an on-site potential $V$ which is absent in the FPU model. Further, we study the case $g<0$, while Calleja and Sire analyse the case $g>0$. In both cases, the existence of solutions homoclinic to exponentially small periodic solutions is proved by combining the setting developed by Iooss and Kirchgässner for lattice waves [7] with the analysis of Lombardi [9] for the existence solutions with oscillatory tails. This commonality rests on the fact that both the FPU chain and the FK chain give rise to simple eigenvalues in the centre spectrum. However, the FPU travelling wave formulation is invariant under translations, since the origin is in the centre spectrum, with multiplicity 4 at the bifurcation point. The origin is not in the centre spectrum for the FK problem, where instead pairs of semisimple eigenvalues exist, unlike for the FPU problem. Consequently, the approach used in the interesting paper [1] does not carry over directly to the system studied here; the central difficulty of the FPU chain is the four-fold multiplicity of the eigenvalue 0 , which is not immediately within the scope of the tools described in Lombardi's book [9]. We use the translational invariance to reduce the multiplicity by one; it can be reduced once more by a normal form analysis, which we carry out in infinite dimensions to retain the analyticity. Then Lombardi's results are directly applicable. The most significant methodological differences between [1] and this paper are 
the treatment of the four-dimensional projection $P_{0}$ in Section 3.1.1, the reduction based on translational invariance in Sections 3.2 and 3.3 , and the infinite-dimensional normal form analysis in Section 3.5. (We are also not aware of results as in Section 4 proved by centre manifold analysis.)

Makita [1] (see also [10]) studies a system which includes some effects of NNN interaction but without competition of the NN and NNN springs mimicking the effect of a Lennard-Jones potential. The physical and numerical literature on NNN models is richer, and we only point the reader to the numerical investigations in [2] and the recent review [13]. Long-range interaction is also relevant in biological systems. For example, to model the three-dimensional helicoidal structure of DNA approximately with a planar description, interactions with fifth neighbours have been proposed [3].

The paper is organised as follows. In Section 2 we describe the general mathematical setting and state the main result (Theorem 1). We also describe the centre spectrum for the linear operator. In Section 3, we concentrate on slightly subsonic wave speeds and show how, for these speeds, the problem can be formulated to use the results in 9 . showing the existence of waves homoclinic to exponentially small (with respect to the bifurcation parameter) periodic waves. In Section 4 , we look at slightly supersonic speeds and sketch the proof of the existence of supersonic periodic solutions.

2. The mathematical setting. We are looking for solutions of the travelling wave equation

$$
\begin{aligned}
c^{2} \ddot{x}(t)=U_{1}^{\prime}(x(t+1)-x(t))- & U_{1}^{\prime}(x(t)-x(t-1)) \\
& +g\left[U_{2}^{\prime}(x(t+2)-x(t))+U_{2}^{\prime}(x(t)-x(t-2))\right] ;
\end{aligned}
$$

here $U_{1}$ is an anharmonic potential for the nearest neighbour interaction and $U_{2}$ is an anharmonic potential for the next-to-nearest neighbour interaction,

$$
U_{1}^{\prime}(r):=c_{0}^{2} r+c^{2} G_{1}^{\prime}(r)
$$

and

$$
U_{2}^{\prime}(r):=c_{0}^{2} r+c^{2} G_{2}^{\prime}(r) .
$$

We remark that $U_{1}$ and $U_{2}$ act against each other since $U_{2}$ is multiplied by $g<0$. For the anharmonic contributions of the potentials,

$$
G_{1}^{\prime}(x):=\alpha_{1} x^{2}+\beta_{1} x^{3}+\ldots \quad \text { and } \quad G_{2}^{\prime}(x):=\alpha_{2} x^{2}+\beta_{2} x^{3}+\ldots,
$$

we assume that

$$
0<\alpha_{2}<\frac{\alpha_{1}}{2}
$$

We also set $\mu:=c_{0}^{2} / c^{2}$. With this form of the interaction potentials, (3) becomes

$$
\begin{aligned}
& \ddot{x}(t)=\mu[x(t+1)-2 x(t)+x(t-1)]+G_{1}^{\prime}(x(t+1)-x(t))-G_{1}^{\prime}(x(t)-x(t-1)) \\
& +\mu g\left\{c_{0}^{2}[x(t+2)-2 x(t)+x(t-2)]+G_{2}^{\prime}(x(t+2)-x(t))-G_{2}^{\prime}(x(t)-x(t-2))\right\} .
\end{aligned}
$$


We are interested in $g$ such that

$$
-\frac{1}{4}<g<-\frac{1}{16}
$$

as, for this range of $g$, the purely harmonic potentials give supersonic periodic solutions with mean zero and the linear operator has a pair of small real eigenvalues for slightly subsonic speeds; see Figure 1], Figure 3 and [15].

Following the method developed by Iooss and Kirchgässner [7, we put $\xi(t):=x^{\prime}(t)$, $X(t, \nu):=x(t+\nu)$ and $U(t)(\nu):=(x(t), \xi(t), X(t, \nu))$, for $\nu \in[-2,2]$. We use the notation $\delta^{i} X(t, \nu):=X(t, i)$ for $i \in\{ \pm 1, \pm 2\}$. Equation (3) can now be written as

$$
\partial_{t} U=L_{\mu, g} U+M_{\mu, g}(U),
$$

where $L_{\mu, g}$ is the linear nonlocal operator

$$
L_{\mu, g}=\left(\begin{array}{ccc}
0 & 1 & 0 \\
-2 \mu(1+g) & 0 & \mu\left(\delta^{1}+\delta^{-1}\right)+\mu g\left(\delta^{2}+\delta^{-2}\right) \\
0 & 0 & \partial_{\nu}
\end{array}\right),
$$

and the nonlinearity is

$$
M_{\mu, g}=\left(0, G_{1}^{\prime}\left(\delta^{1} X-x\right)-G_{1}^{\prime}\left(x-\delta^{-1} X\right)+g\left(G_{2}^{\prime}\left(\delta^{2} X-x\right)-G_{2}^{\prime}\left(x-\delta^{-2} X\right)\right), 0\right)^{T} .
$$

The boundary condition is then

$$
X(t, 0)=x(t) .
$$

We use a functional setting as in [7] and thus define the Banach spaces $\mathbb{H}$ and $\mathbb{D}$ for $U(\nu)$ (here and below, we often suppress a functional dependence on $t$ ) as

$$
\mathbb{H}:=\mathbb{R}^{2} \times\left(C^{0}[-2,2]\right) \quad \text { and } \quad \mathbb{D}:=\left\{U \in \mathbb{R}^{2} \times\left(C^{1}[-2,2]\right) \mid X(0)=x\right\}
$$

endowed with the usual maximum norms. As in [7, the operator $L_{\mu, g}$ maps $\mathbb{D}$ continuously into $\mathbb{H}$ and has a compact resolvent in $\mathbb{H}$ when acting in $\mathbb{H}$ with domain $\mathbb{D}$.

The nonlinearity $M_{\mu, g}$ is assumed to be analytic in $t$ and $\mu$. This implies that, for any $\rho>0$, there is a $C(\rho)$ such that $\left\|M_{\mu, g}(U)\right\| \leq C(\rho)\|U\|_{\mathbb{D}}^{2}$ for $\|U\|_{\mathbb{D}} \leq \rho$. Analyticity is a much stronger assumption than Iooss makes in [5], but it is needed to enable us to use the results developed by Lombardi in 9 .

The main result of this article is as follows.

Theorem 1. Suppose the potentials satisfy (44)-(7), and the nonlinearity $M_{\mu, g}$ as in (10) is analytic. For $g$ with $-\frac{1}{4}+\frac{1}{4 \pi^{2}}<g<-\frac{1}{16}$, there exists $\hat{c}<c_{0}(1+4 g)$ such that, for $c \in\left(\hat{c}, c_{0}(1+4 g)\right)$, equation (3) has a solution comprising of a travelling wave with speed $c$ which is homoclinic to exponentially small periodic solutions of (3).

The theorems used from [9] could be used to give estimates on the amplitude of the periodic solutions in Theorem 1. We concentrate, however, on showing how the problem can be formulated so that the work in [9] is applicable to the NNN lattice and hence prove the existence of the waves described in Theorem 1 


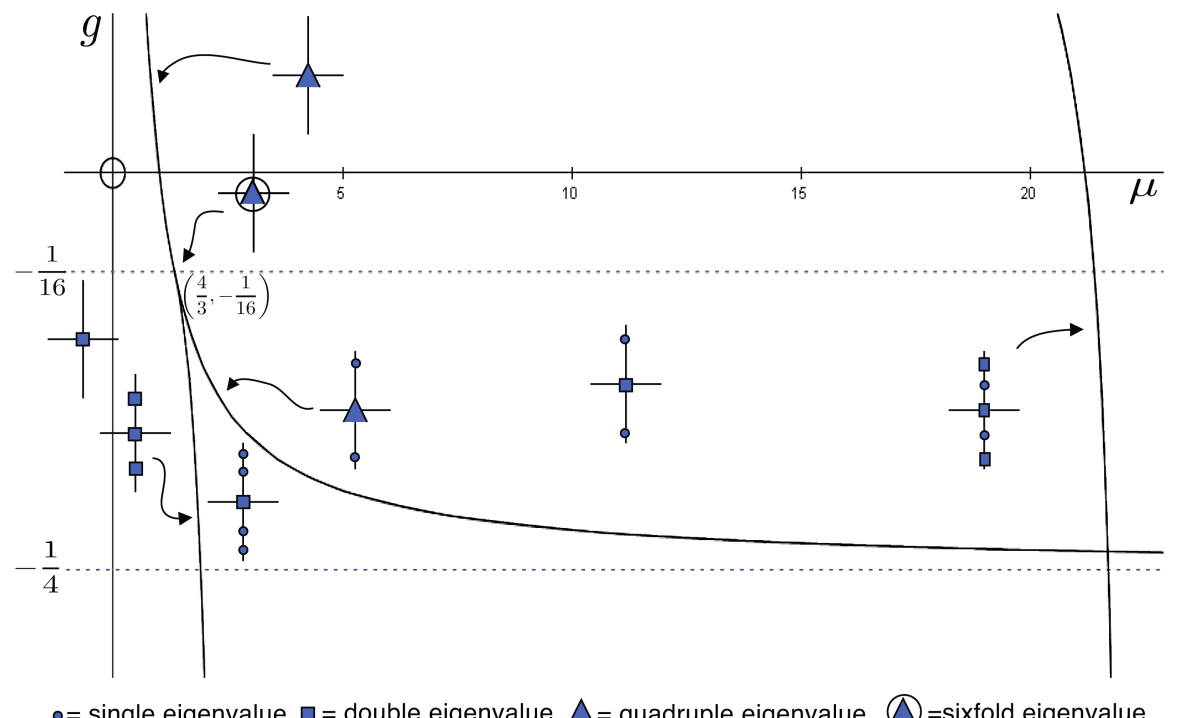

FIG. 1. The bifurcation diagram for the problem considered in this article.

The upper bound on the wave speed in Theorem 1 can be rephrased as the condition $\mu>\frac{1}{1+4 g}$. The corresponding spectrum is shown in Figure 3 at the bottom, rightmost plot. We remark that the speed with which waves can propagate in the linearisation of (8) is $c=c_{0} \sqrt{1+4 g}$, which is equivalent to

$$
\mu=\frac{1}{1+4 g} .
$$

We call $c_{0} \sqrt{1+4 g}$ the speed of sound and, consequently, Theorem 1 establishes the existence of subsonic waves. Our terminology follows, for example, [16], and refers to the speed of waves in the linearised model. We point out that sometimes the group velocity is called speed of sound.

Equation (3), like the NN systems of [5, 7], is invariant under the symmetry $t \rightarrow-t$, $x \rightarrow-x$. Thus $L_{\mu, g}$ and $M_{\mu, g}$ both anti-commute with the reflection $S$ in $\mathbb{H}$ given by $S(x, \xi, X)^{T}=(-x, \xi,-X \circ s)^{T}$, where $S(x, \xi, X(\nu))^{T}=(-x, \xi,-X(-\nu))^{T}$; anticommutativity means

$$
L_{\mu, g} S=-S L_{\mu, g} \quad \text { and } \quad M_{\mu, g} S=-S M_{\mu, g} .
$$

Also, we notice that, as in [6], the equation has a first integral

$$
I_{1}=c^{2} \dot{x}(t)-\int_{0}^{1} U_{1}^{\prime}(x(t+\nu)-x(t+\nu-1)) d \nu-g \int_{0}^{2} U_{2}^{\prime}(x(t+\nu)-x(t+\nu-2)) d \nu .
$$

2.1. The spectrum and resolvent of $L_{\mu, g}$. In this section, we determine the spectrum $\Sigma$ of $L_{\mu, g}$ by solving the equation

$$
\left(\lambda \mathbb{I}-L_{\mu, g}\right) U=F
$$


for any given $F=\left(f_{0}, f_{1}, F_{2}\right)^{T} \in \mathbb{H}$; here $\lambda \in \mathbb{C}$ and $U=(x, \xi, X)^{T} \in \mathbb{D}$. Again, we often suppress the time-dependence of the variables involved by writing, for example, $x$ instead of $x(t)$.

The third equation of (15) can be solved for $X(\nu)$ by variation of constants; terms involving $X(\nu)$ in the first two equations can then be calculated and these two equations can be solved simultaneously for $x$ and $\xi$. We express the solution in terms of

$$
N(\lambda ; \mu, g):=-\lambda^{2}+2 \mu(\cosh \lambda-1)+2 \mu g(\cosh 2 \lambda-1)
$$

and

as

$$
\tilde{f}_{\lambda_{j}}:=\int_{0}^{j}\left[-e^{\lambda(j-s)} F_{2}(s)+e^{-\lambda(j-s)} F_{2}(-s)\right] d s
$$

$$
\begin{aligned}
x & =-(N(\lambda ; \mu, g))^{-1}\left[\lambda f_{0}+f_{1}+\mu\left(\tilde{f}_{\lambda_{1}}+g \tilde{f}_{\lambda_{2}}\right)\right], \\
\xi & =-(N(\lambda ; \mu, g))^{-1}\left[\left(\lambda^{2}+N(\lambda ; \mu, g)\right) f_{0}+\lambda f_{1}+\mu \lambda\left(\tilde{f}_{\lambda_{1}}+g \tilde{f}_{\lambda_{2}}\right)\right], \\
X(\nu) & =e^{\lambda \nu} x-\int_{0}^{\nu} e^{\lambda(\nu-s)} F_{2}(s) d s .
\end{aligned}
$$

These formulae hold provided $N(\lambda ; \mu, g) \neq 0$. For each $(\mu, g) \in \mathbb{R}^{2}, N(\lambda ; \mu, g)$ is an entire function in $\lambda$, and thus has isolated zeros of finite multiplicities. Therefore the spectrum of $L_{\mu, g}$ consists of isolated eigenvalues with finite multiplicities located at these zeros. Also, as in [5], the spectrum is invariant under reflexions in the real and imaginary axes, which follows from (13). We thus can confine the examination to $\lambda=p+i q$, where $p$ and $q$ are positive.

For the central part $\Sigma_{0}$ of the spectrum, $N(i q ; \mu, g)=0$ with $q \in \mathbb{R}$, i.e.,

$$
q^{2}=2 \mu[(1-\cos q)+g(1-\cos 2 q)]
$$

or, equivalently,

$$
\frac{q}{2 \sqrt{\mu}}=\left|\sin \frac{q}{2} \sqrt{1+4 g \cos ^{2} \frac{q}{2}}\right| .
$$

We recall the assumption $-\frac{1}{4}<g<-\frac{1}{16}$, when (22) can be illustrated as in Figure 2 , To keep the bifurcation at $\mu=\frac{1}{1+4 g}$ as simple as possible, we further restrict the values of $g$ under consideration, so that there are no more than two solutions to (22); the right panel in Figure 2 suggests that a very safe restriction is to stipulate that $\frac{1}{\pi}<\sqrt{1+4 g}$, i.e., $g>-\frac{1}{4}+\frac{1}{4 \pi^{2}}$. (This lower bound for $g$ could be improved by more detailed calculations.)

The following lemma summarises central spectral properties of $L_{\mu, g}$.

Lemma 1. If $-\frac{1}{4}+\frac{1}{4 \pi^{2}}<g<-\frac{1}{16}$, the spectrum for $L_{\mu, g}$ has the following properties.

(1) Spectral gap: For each $(\mu, g) \in \mathbb{R}^{+} \times \mathbb{R}$, there exists $p_{0}>0$ such that for every $\lambda \in \Sigma L_{\mu, g} \backslash \Sigma_{0},|\operatorname{Re}(\lambda)| \geq p_{0}$.

(2) If $\lambda=p+i q \in \Sigma \backslash \Sigma_{0}$, then

$$
q^{2} \leq 4\left(\mu(1+|g|)+e^{-2}\right) \cosh ^{2} p-4 \mu|g| .
$$

(3) There exists $\mu_{g}$ with $0<\mu_{g}<\frac{1}{1+4 g}$ such that for $0<\mu<\mu_{g}$, 0 is the only eigenvalue in the central spectrum and has multiplicity 2 . There are two pairs of complex eigenvalues which converge towards the imaginary axis as $\mu \rightarrow \mu_{g}$. 


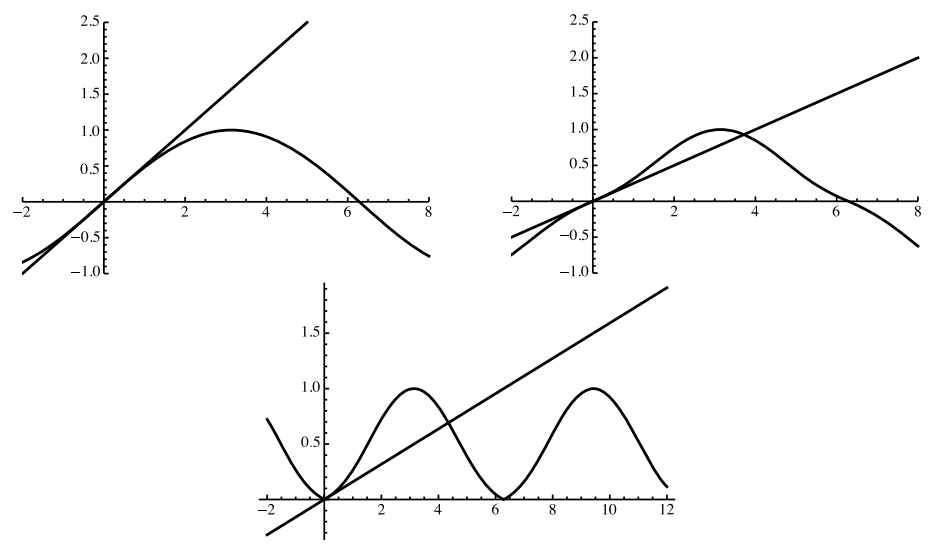

FIG. 2. Illustration of the dispersion function for NN interaction on the left and NNN interaction in the middle and on the right. Shown are plots of $\frac{1}{\sqrt{\mu}} \frac{q}{2}$ and $\sin \left(\frac{q}{2}\right) \sqrt{1+4 g \cos ^{2}\left(\frac{q}{2}\right)}$, for $\mu$ as in (12), $g=0$ (upper left), $g=-\frac{3}{16}$ (upper right); the lower plot shows the same curves except that the modulus is taken for the nonlinear function, and $g=-\frac{1}{4}+\frac{1}{4 \pi^{2}}$.

At $\mu=\mu_{g}, 0$ is an eigenvalue of multiplicity 2 and there is also a pair $\pm i q_{0}$ of double, non semi-simple eigenvalues.

For $\mu_{g}<\mu<\frac{1}{1+4 g}, 0$ is an eigenvalue of multiplicity 2, and there are two pairs of simple eigenvalues $\pm i q_{1}$ and $\pm i q_{2}$.

At $\mu=\frac{1}{1+4 g}, 0$ is an eigenvalue of multiplicity 4 , and there is a pair of simple imaginary eigenvalues $\pm i q_{2}$.

For $\mu$ just greater than $\frac{1}{1+4 g}$, there is a double eigenvalue at zero, a pair of simple imaginary eigenvalues, $\pm i q_{2}$, and two real eigenvalues which then move away from zero as $\mu$ increases.

See Figure 3 for an illustration.

(4) There is then an unbounded sequence of critical values of $\mu$ at which bifurcations occur and the dimension of the centre space increases by 4 .

Proof. The proof follows the method in Iooss and Kirchgässner [7, Lemma 1], and we omit the proofs for Claims 1 and 2

For Claims 3 and 4, we note that

$$
N(i q ; \mu, g)=q^{2}-2 \mu(1-\cos q)-2 \mu g(1-\cos 2 q)=q^{2}-4 \mu \sin ^{2} \frac{q}{2}-4 \mu g \sin ^{2} q,
$$

and since

$$
\frac{\partial N(i q ; \mu, g)}{\partial q}=2 q-2 \mu \sin q-4 \mu g \sin 2 q,
$$

it follows that for every $(\mu, g)$ the eigenvalue 0 has multiplicity of at least 2. Similarly,

$$
\frac{\partial^{2} N(i q ; \mu, g)}{\partial q^{2}}=2-2 \mu \cos q-8 \mu g \cos 2 q \quad \text { and } \quad \frac{\partial^{3} N(i q ; \mu, g)}{\partial q^{3}}=2 \mu \sin q+16 \mu g \sin 2 q
$$




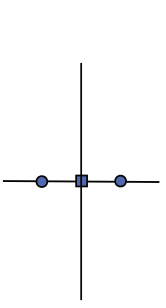

$\mu<1$

$$
g=0
$$

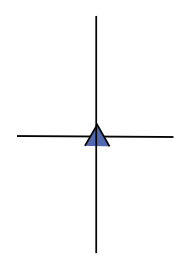

$\mu=1$

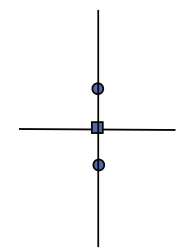

$\mu>1$

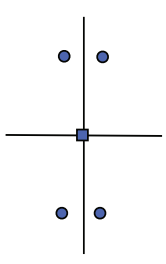

$\mu<\mu_{g}$

$$
-\frac{1}{4}+\frac{1}{4 \pi^{2}}<g<-\frac{1}{16}
$$
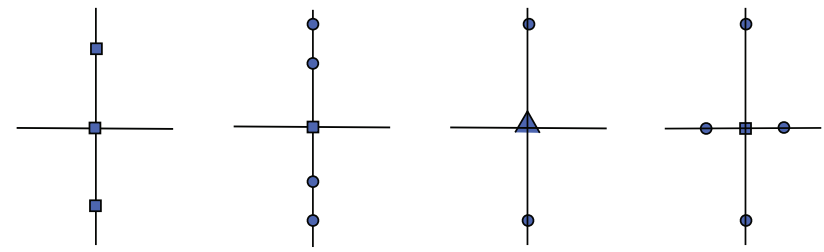

$$
\mu=\mu_{g}
$$

$$
\mu_{g}<\mu<\frac{1}{1+4 g} \quad \mu=\frac{1}{1+4 g}
$$

$\mu>\frac{1}{1+4 g}$

FIG. 3. Eigenvalues at the bifurcation $\mu=\frac{1}{1+4 g}$ in $\mathbb{C}$ (bottom) and bifurcation for the NN case $(g=0$, top). Circles denote single eigenvalues, squares double eigenvalues and a triangle an eigenvalue of multiplicity 4 .

so for a pair $(\mu, g)$, if the eigenvalue 0 has multiplicity greater than 2 , it has multiplicity of at least 4 , and such pairs satisfy $\mu=\frac{1}{1+4 g}$. Moreover, one infers analogously from $\frac{\partial^{4} N}{\partial q^{4}}$ and $\frac{\partial^{5} N}{\partial q^{5}}$ that the multiplicity of 0 is 6 if $\mu=1 /(1+4 g)$ and $g=-1 / 16$, that is, $(\mu, g)=(4 / 3,-1 / 16)$.

Setting (23) and (24) to 0 and eliminating $g$ gives

$$
\mu=\frac{q(\sin q-q \cos q)}{(1-\cos q)^{2}}
$$

substituting this into (24) gives

$$
g=\frac{q}{4(\sin q-q \cos q)}-\frac{\sin q}{2(1+\cos q)(\sin q-q \cos q)} .
$$

These two equations parametrise the curve shown in Figure 3.

3. The bifurcation at $\mu=1 /(1+4 g)$ and the existence of subsonic travelling waves homoclinic to periodic solutions. We keep the analysis confined to the case $g \in\left(-\frac{1}{4}+\frac{1}{4 \pi^{2}},-\frac{1}{16}\right)$; then, by Lemma 1, the linear operator $L_{\mu, g}$ has a eigenvalue of multiplicity 4 at 0 and a pair of simple eigenvalues, now simply referred to as $\pm i q$.

3.1. The projection onto the centre space for the linear operator. 
3.1.1. The projection $P_{0}$. Let $P_{0}$ be the projection onto the space spanned by the Jordan basis

$$
\zeta_{0}=\left(\begin{array}{l}
1 \\
0 \\
1
\end{array}\right), \quad \zeta_{1}=\left(\begin{array}{c}
0 \\
1 \\
\nu
\end{array}\right), \quad \zeta_{2}=\left(\begin{array}{c}
0 \\
0 \\
\frac{1}{2} \nu^{2}
\end{array}\right), \quad \zeta_{3}=\left(\begin{array}{c}
0 \\
0 \\
\frac{1}{6} \nu^{3}
\end{array}\right) .
$$

Note that, for $\mu=1 /(1+4 g)$,

$$
\begin{aligned}
& L_{\mu, g} \zeta_{0}=0 \quad \text { and } \quad S \zeta_{0}=-\zeta_{0} \\
& L_{\mu, g} \zeta_{1}=\zeta_{0} \quad \text { and } \quad S \zeta_{1}=\zeta_{1} \\
& L_{\mu, g} \zeta_{2}=\zeta_{1} \text { and } S \zeta_{2}=-\zeta_{2} \\
& L_{\mu, g} \zeta_{3}=\zeta_{2} \text { and } S \zeta_{3}=\zeta_{3} \text {. }
\end{aligned}
$$

We proceed as in Iooss [6] and use that the projection is given by the Laurent expansion in $\mathbb{L}(\mathbb{H})$ of the resolvent operator, $\left(\lambda \mathbb{I}-L_{\mu, g}\right)^{-1}$, for $\lambda$ near 0 . So if $D:=L_{\mu, g} P_{0}$ and $\tilde{L}_{\mu, g}^{-1}$ is the pseudo-inverse of $L_{\mu, g}$ on the subspace $\left(\mathbb{I}-P_{0}\right) \mathbb{H}$, then [8, Chapter III, Eq. (6.32)]

$$
\left(\lambda \mathbb{I}-L_{\mu, g}\right)^{-1}=\frac{D^{3}}{\lambda^{4}}+\frac{D^{2}}{\lambda^{3}}+\frac{D}{\lambda^{2}}+\frac{P_{0}}{\lambda}-\tilde{L}_{\mu, g}^{-1}-\lambda \tilde{L}_{\mu, g}^{-2}-\ldots,
$$

and, if $F=\left(f_{0}, f_{1}, F_{2}\right)^{T} \in \mathbb{H}$,

$$
P_{0} F=\left(\left(P_{0} F\right)_{x},\left(P_{0} F\right)_{\xi},\left(P_{0} F\right)_{X}\right)^{T}=\left(P_{0} F\right)_{x} \zeta_{0}+(D F)_{x} \zeta_{1}+\left(D^{2} F\right)_{x} \zeta_{2}+\left(D^{3} F\right)_{x} \zeta_{3} .
$$

We expand $\left(N\left(\lambda ; \frac{1}{1+4 g}, g\right)\right)^{-1}$ and $\tilde{f}_{\lambda_{j}}$ for $j=1,2$ in powers of $\lambda$ and compare powers of $\lambda$ in (18).

The coefficient of $\lambda^{-4}$ gives

$$
\begin{aligned}
\left(D^{3} F\right)_{x} & =\left(D^{2} F\right)_{\xi} \\
& =-\frac{12}{1+16 g}\left\{(1+4 g) f_{1}-\int_{0}^{1}\left[F_{2}(s)-F_{2}(-s)\right] d s-g \int_{0}^{2}\left[F_{2}(s)-F_{2}(-s) d s\right]\right\} .
\end{aligned}
$$

From the coefficient of $\lambda^{-3}$, we obtain

$$
\begin{aligned}
\left(D^{2} F\right)_{x}=(D F)_{\xi}= & \frac{-12}{1+16 g}\left\{(1+4 g) f_{0}-\int_{0}^{1}(1-s)\left(F_{2}(s)+F_{2}(-s)\right) d s\right. \\
& \left.-g \int_{0}^{2}(2-s)\left(F_{2}(s)+F_{2}(-s)\right) d s\right\},
\end{aligned}
$$

while the coefficient of $\lambda^{-2}$ gives

$$
\begin{aligned}
(D F)_{x} & =(P F)_{\xi} \\
& =\frac{12}{1+16 g}\left\{\frac{(1+64 g)(1+4 g)}{30(1+16 g)} f_{1}-\int_{0}^{1}\left(\frac{1+64 g}{30(1+16 g)}-\frac{(1-s)^{2}}{2}\right)\right. \\
& \left.\cdot\left(F_{2}(s)-F_{2}(-s)\right) d s-g \int_{0}^{2}\left(\frac{1+64 g}{30(1+16 g)}-\frac{(2-s)^{2}}{2}\right)\left(F_{2}(s)-F_{2}(-s)\right) d s\right\} ;
\end{aligned}
$$


and finally, from the coefficient of $\lambda^{-1}$,

$$
\begin{aligned}
(P F)_{x}= & \frac{2}{5(1+16 g)}\left\{\frac{(1+64 g)(1+4 g)}{(1+16 g)} f_{0}\right. \\
& -\int_{0}^{1}\left(\frac{(1+64 g)(1-s)}{(1+16 g)}-5(1-s)^{3}\right)\left(F_{2}(s)+F_{2}(-s)\right) d s \\
& \left.-g \int_{0}^{2}\left(\frac{(1+64 g)(2-s)}{(1+16 g)}-5(2-s)^{3}\right)\left(F_{2}(s)+F_{2}(-s)\right) d s\right\} .
\end{aligned}
$$

As in Iooss' paper [5], we denote by $\zeta_{j}^{*}$ the linear continuous forms on $\mathbb{H}$ given by $\zeta_{0}^{*}(F)=(P F)_{x}, \zeta_{1}^{*}(F)=(D F)_{x}=\zeta_{0}^{*}\left(L_{\mu, g} F\right), \zeta_{2}^{*}(F)=\left(D^{2} F\right)_{x}$ and $\zeta_{3}^{*}(F)=\left(D^{3} F\right)_{x}$. It holds that

$$
\begin{aligned}
\zeta_{j}^{*}(S F) & =(-1)^{j+1} \zeta_{j}^{*}(F) \\
\zeta_{k}^{*}\left(\zeta_{j}\right) & =\delta_{k j} \quad \text { for } \quad k, j=0,1,2,3 .
\end{aligned}
$$

We also notice that, if $F=W=(x, \xi, X(v))^{T} \in \mathbb{D}$, then (14) gives us

$$
\begin{aligned}
I_{1}= & c^{2} \xi-c^{2} \mu \int_{0}^{1}[x(t+\nu)-x(t+\nu-1)] d \nu \\
& \quad-c^{2} \mu g \int_{0}^{2}[x(t+\nu)-x(t+\nu-2)] d \nu+\text { h.o.t. } \\
= & c^{2} \xi-c^{2} \mu \int_{0}^{1}[X(\nu)-X(\nu-1)] d \nu-c^{2} \mu g \int_{0}^{2}[X(\nu)-X(\nu-2)] d \nu+\text { h.o.t. } \\
= & c^{2} \xi-c^{2} \mu \int_{0}^{1}[X(\nu)-X(-\nu)] d \nu-c^{2} \mu g \int_{0}^{2}[X(\nu)-X(-\nu)] d \nu+\text { h.o.t. } \\
= & -\frac{c^{2} \mu(1+16 g)}{12} \zeta_{3}^{*}(W)+\text { h.o.t. }
\end{aligned}
$$

So we can write

$$
\zeta_{3}^{*}(W)=C+\text { h.o.t. , }
$$

where $C$ is a constant and can be regarded as a parameter of the system.

3.1.2. The projection $P_{q}$. Let $P_{q}$ be the projection on the invariant subspace spanned by

$$
\zeta_{q}=\left(\begin{array}{c}
1 \\
i q \\
e^{i q}
\end{array}\right) \text { and } \bar{\zeta}_{q}=\left(\begin{array}{c}
1 \\
-i q \\
e^{-i q}
\end{array}\right)
$$

We define, as in [7], for $j=1,2$,

$$
\begin{aligned}
\sigma_{j}(F) & :=\int_{0}^{j} \sin q(j-s)\left[F_{2}(s)+F_{2}(-s)\right] d s, \\
\rho_{j}(F) & :=\int_{0}^{j} \cos q(j-s)\left[F_{2}(s)-F_{2}(-s)\right] d s .
\end{aligned}
$$


The projection $P_{q}$ is then given by the sum of the residues at $\pm i q$ for the three components in (18)-(20). That is,

$$
\begin{aligned}
& \left(P_{q} F\right)_{0}=\frac{q f_{0}-\mu \sigma_{1}-\mu g \sigma_{2}}{N_{1}}, \\
& \left(P_{q} F\right)_{1}=\frac{q f_{1}-q \mu\left(\rho_{1}+g \rho_{2}\right)}{N_{1}},
\end{aligned}
$$

and

$$
\left(P_{q} F\right)_{2}=\frac{\cos q \nu\left(q f_{0}-\mu\left(\sigma_{1}+g \sigma_{2}\right)\right)+\sin q \nu\left(f_{1}-\mu\left(\rho_{1}+g \rho_{2}\right)\right)}{N_{1}} .
$$

Straightforward calculations show that

$$
\begin{aligned}
& P_{q} \zeta_{q}=\zeta_{q}, \\
& P_{0} \zeta_{j}=\zeta_{j} \quad \text { for } \quad j=0,1,2,3 \text {, } \\
& P_{0} \zeta_{q}=0 \text {, } \\
& P_{q} \zeta_{j}=0 \quad \text { for } \quad j=0,1,2,3 \text {. }
\end{aligned}
$$

3.2. Reduction of the system. As for the system with NN interaction only [5], the system (9) is invariant under the shift operator

$$
\tau_{r}: U \longmapsto U+r \zeta_{0} \quad \text { for all } \in \mathbb{R},
$$

which corresponds to the invariance of (3) under $x \longmapsto x+r$. Invariance means here

$$
L_{\mu, g} \tau_{r}=L_{\mu, g} \quad \text { and } \quad M_{\mu, g} \circ \tau_{r}=M_{\mu, g} .
$$

So we decompose any $U \in \mathbb{H}$ into

$$
U=: W+r(t) \zeta_{0} \quad \text { with } \quad \zeta_{0}^{*}(W)=0 ;
$$

for the corresponding split of the function spaces, let us write $\hat{H}$ for the co-dimension-one subspace of $\mathbb{H}$ containing $W$ and $\hat{\mathbb{D}}$ for the corresponding subspace of $\mathbb{D}$.

Since $M_{\mu, g}$ has the form $\left(0, f_{1}, 0\right), \zeta_{0}^{*}\left(M_{\mu, g}\right)=0$, so (9) becomes the system

$$
\begin{aligned}
\frac{\partial r}{\partial t} & =\zeta_{0}^{*}\left(L_{\mu, g} W\right)+0=\zeta_{1}^{*}(W), \\
\frac{\partial W}{\partial t} & =\hat{L}_{\mu, g} W+M_{\mu, g}(W),
\end{aligned}
$$

where

and we still have $S \hat{L}_{\mu, g}=-\hat{L}_{\mu, g} S$.

$$
\hat{L}_{\mu, g} W:=L_{\mu, g} W-\zeta_{1}^{*}(W) \zeta_{0}
$$

We note that $\hat{L}_{\mu, g}$ has the same spectrum as $L_{\mu, g}$ except that the eigenvalue at 0 is triple rather than quadruple and

$$
\hat{L}_{\mu, g} \zeta_{1}=0, \quad \hat{L}_{\mu, g} \zeta_{2}=\zeta_{1}, \quad \hat{L}_{\mu, g} \zeta_{3}=\zeta_{2}, \quad \zeta_{3}^{*}\left(\hat{L}_{\mu, g} W\right)=0 .
$$

We now wish to deduce the normal form for $\hat{L}_{\mu, g}$. However, so that we can use the results in Lombardi's book [9], we do not carry out a centre manifold reduction but rather deduce a normal form in infinite dimensions, so that analyticity is retained. 
We study the equation (39) in the Banach space $\hat{\mathbb{H}}$. We want to study $\mu$ close to $1 /(1+4 g)$ for a fixed $g \in\left(-\frac{1}{4}+\frac{1}{4 \pi^{2}},-\frac{1}{16}\right)$. We thus define the parameter $\psi:=\mu-$ $1 /(1+4 g)$ and consider $\psi \in\left[-\psi_{0}, \psi_{0}\right]$ for some $\psi_{0}>0$. The equation is now sometimes written in the form

$$
\frac{\partial W}{\partial t}=\hat{L}_{\mu, g}(\psi) W+M_{\mu, g}(W, \psi) .
$$

We split the linear part as

$$
\hat{L}_{\mu, g}(\psi)=\hat{L}^{0}+\psi \hat{L}^{1}
$$

where $\hat{L}^{0}$ denotes the operator

$$
\hat{L}^{0}:=\hat{L}_{\frac{1}{1+4 g}, g}=\left(\begin{array}{ccc}
0 & 1 & 0 \\
-2 \frac{(1+g)}{(1+4 g)} & 0 & \frac{\delta^{1}+\delta^{-1}+g\left(\delta^{2}+\delta^{-2}\right)}{(1+4 g)} \\
0 & 0 & \partial_{\nu}
\end{array}\right)-\zeta_{1}^{*}(.) \zeta_{0},
$$

and the operator

$$
\hat{L}^{1}:=\left(\begin{array}{ccc}
0 & 0 & 0 \\
-2(1+g) & 0 & \left(\delta^{1}+\delta^{-1}\right)+g\left(\delta^{2}+\delta^{-2}\right) \\
0 & 0 & 0
\end{array}\right)
$$

is independent of $\psi$.

The central spectrum $\Sigma_{0}=\Sigma \hat{L}^{0} \cap i \mathbb{R}$ of $\hat{L}^{0}$ is $\{ \pm i q, 0\}$ with $q>0 ; 0$ is a triple non semi-simple isolated eigenvalue, and $\pm i q$ are two simple isolated eigenvalues.

Since the spectrum suggests that there are homoclinics to exponentially small oscillatory tails, we would like to apply the results of Lombardi's book 9]. However, there are two obstacles. Firstly, 0 is a double rather than a triple eigenvalue in [9. We will deal with this by establishing a normal form with one coordinate as a constant (the constant of integration established in (29) ) and then we can effectively factor out $\zeta_{3}$ and thus further reduce the multiplicity of the eigenvalue 0 to 2 .

Secondly, in [9], it is assumed that the spectrum of $\hat{L}^{0}$ is sectorial. This, in turn, implies that the hyperbolic part of the system can be solved uniquely. This solvability property is a key requirement for the proof of persistence of solutions for the full equation. In our case, the spectrum is not sectorial; see Lemma 1 (for $L_{\mu, g}$; the same is true for $\hat{L}_{\mu, g}$ ). We circumvent this difficulty by showing that the hyperbolic part of the system can still be solved uniquely in an appropriate space; the argument uses the ideas in Iooss and Kirchgässner [7].

3.3. Properties of $\hat{L}_{\mu, g}$. We define the graph norm on $\hat{\mathbb{D}}$ as

$$
\|W\|_{\hat{\mathbb{D}}}^{2}:=\|W\|_{\hat{\mathbb{H}}}^{2}+\left\|\hat{L}^{0} W\right\|_{\hat{\mathbb{H}}}^{2} .
$$

We now consider the centre spaces in $\mathbb{H}$ (respectively $\hat{H})$. In $\mathbb{H}$, we denote the finitedimensional space spanned by $\left\{\zeta_{0}, \zeta_{1}, \zeta_{2}, \zeta_{3}, \zeta_{q}, \zeta_{-q}\right\}$ by $\mathbb{H}_{c}$ and the subspace of $\hat{\mathbb{H}}$ spanned by $\left\{\zeta_{1}, \zeta_{2}, \zeta_{3}, \zeta_{q}, \zeta_{-q}\right\}$ as $\hat{\mathbb{H}}_{c}$. For the projection onto $\mathbb{H}_{c}$ we write

$$
P_{c} U:=\left(\zeta_{0}^{*}, U\right) \zeta_{0}+\left(\zeta_{1}^{*}, U\right) \zeta_{1}+\left(\zeta_{2}^{*}, U\right) \zeta_{2}+\left(\zeta_{3}^{*}, U\right) \zeta_{3}+\left(\zeta_{q}^{*}, U\right) \zeta_{q}+\left(\zeta_{-q}^{*}, U\right) \zeta_{-q},
$$

and the projection on $\hat{\mathbb{H}}_{c}$ is

$$
\hat{P}_{c} W:=\left(\zeta_{1}^{*}, W\right) \zeta_{1}+\left(\zeta_{2}^{*}, W\right) \zeta_{2}+\left(\zeta_{3}^{*}, W\right) \zeta_{3}+\left(\zeta_{q}^{*}, W\right) \zeta_{q}+\left(\zeta_{-q}^{*}, W\right) \zeta_{-q} .
$$


It is trivial to check that these operators satisfy $P_{c} \in \mathbb{L}(\mathbb{H}, \mathbb{D})$ and $\hat{P}_{c} \in \mathbb{L}(\hat{\mathbb{H}}, \hat{\mathbb{D}})$. The projection $\hat{P}_{c}$ also commutes with $\hat{L}^{0}$; that is, $\hat{L}^{0} \hat{P}_{c} W=\hat{P}_{c} \hat{L}^{0} W$, for all $W \in \hat{\mathbb{D}}$.

The projection of $\mathbb{H}$ on the hyperbolic part is denoted $P_{h}:=\mathrm{Id}-P_{c}$; we write $\mathbb{H}_{h}:=$ $P_{h} \mathbb{H}$ for the range. Similarly, let us define the projection $\hat{P}_{h}:=\mathrm{Id}-\hat{P}_{c}$ acting on $\hat{\mathbb{H}}$. Note that $\hat{P}_{h} \hat{\mathbb{H}}=\mathbb{H}_{h}$ since the difference between $\mathbb{H}$ and $\hat{\mathbb{H}}$ is in the centre space.

Similarly, let $\mathbb{D}_{h}:=P_{h} \mathbb{D}=\hat{P}_{h} \hat{\mathbb{D}}$ and define the restricted operators $\hat{L}_{c}^{0}:=\left.\hat{L}^{0}\right|_{\hat{\mathbb{H}}_{c}}$ and $\hat{L}_{h}^{0}:=\left.\hat{L}^{0}\right|_{\mathbb{H}_{h}}$.

Then the split in centre and hyperbolic space can be written as $\mathbb{H}=\mathbb{H}_{c} \oplus \mathbb{H}_{h}$ and $\mathbb{D}=\mathbb{D}_{c} \oplus \mathbb{D}_{h} ;$ analogously, $\hat{\mathbb{H}}=\hat{\mathbb{H}}_{c} \oplus \mathbb{H}_{h}$ and $\hat{\mathbb{D}}=\hat{\mathbb{D}}_{c} \oplus \mathbb{D}_{h}$.

In the following, we identify $\hat{\mathbb{H}}$ with $\mathbb{R}^{5} \times \mathbb{H}_{h}$ and $\hat{\mathbb{D}}$ with $\mathbb{R}^{5} \times \mathbb{D}_{h}$. Then (41) can be written as

$$
\begin{aligned}
\frac{\partial W_{c}}{\partial t} & =\hat{L}_{c}^{0} W_{c}+M_{c}(W, \psi) \\
\frac{\partial W_{h}}{\partial t} & =\hat{L}_{h}^{0} W_{h}+M_{h}(W, \psi),
\end{aligned}
$$

where $M_{c}(W, \psi):=\psi \hat{P}_{c} \hat{L}^{1}(\psi) W+\hat{P}_{c} M_{\mu, g}(W, \psi)$ and $M_{h}(W, \psi):=\psi \hat{P}_{h} \hat{L}^{1}(\psi) W+$ $\hat{P}_{h} M_{\mu, g}(W, \psi)$. We have suppressed the subscript " $\mu, g$ " from the restricted operators for better readability. We now collect some properties of the operators involved.

LEMma 2. (1) $\hat{L}(\psi)$ is a closed, densely defined, unbounded linear operator in $\hat{\mathbb{H}}$, with the domain $\hat{\mathbb{D}}$ being independent of $\psi$.

(2) The system (41) is reversible: there exists a reflection $S$, that is, a bounded linear operator in $\mathbb{H}_{H}$ satisfying $S^{2}=\operatorname{Id}_{\hat{\mathbb{H}}}$, such that $S \hat{L}_{\mu, g}(\psi) W=-\hat{L}_{\mu, g}(\psi) S W$ and $S M_{\mu, g}(W, \psi)=-M_{\mu, g}(S W, \psi)$ for every $W \in \hat{\mathbb{D}}$ and $\psi \in\left[-\psi_{0}, \psi_{0}\right]$.

(3) There exists a constant $C_{L}$ such that

$$
\left\|\hat{L}^{1}(\psi) W\right\|_{\hat{\mathbb{H}}} \leq C_{L}\|W\|_{\hat{\mathbb{D}}}
$$

for every $\psi \in\left[-\psi_{0}, \psi_{0}\right]$ and $W \in \hat{\mathbb{D}}$.

(4) (a) The nonlinearity $M_{\mu, g}(W, \psi)$ is an analytic function from $B(0, r) \times\left[-\psi_{0}, \psi_{0}\right]$ to $\hat{\mathbb{H}}$, where $B(0, r)$ is some ball of radius $r$ in $\hat{\mathbb{D}}$.

(b) There exists a constant $C_{M_{\mu, g}}$ such that

$$
\left\|M_{\mu, g}(W, \psi)\right\|_{\hat{\mathbb{H}}} \leq C_{M_{\mu, g}}\|W\|_{\hat{\mathbb{D}}}^{2}
$$

for every $W$ in $B(0, r)$, and $\psi \in\left[-\psi_{0}, \psi_{0}\right]$.

Proof. 1 follows directly from the definitions; both 2 and 4 are inherited from the original system defined on $\mathbb{H}$. For example, 2 is the restriction of (13) to $\hat{L}_{\mu, g}$. As for 3 , (45) follows with $C_{L}:=4(1+|g|)$ from (42).

We remark that properties 2 and 4 ensure that necessarily $S \zeta_{1}= \pm \zeta_{1}$. We actually have $S \zeta_{1}=\zeta_{1}$; i.e., in the notation of Lombardi [9], we have a $0^{3+} i q$ resonance at the origin. 
3.4. Solving the hyperbolic equation. We employ the spaces $E_{j}^{\alpha}(Z)$ of [7] for $\alpha \in \mathbb{R}$ and $j \in \mathbb{N}$, with norms $\|f\|_{j}$,

$$
E_{j}^{\alpha}(Z)=\left\{f \in C^{j}(\mathbb{R}, Z)\left|\|f\|_{j}=\max _{0 \leq k \leq j} \sup _{t \in \mathbb{R}} e^{-\alpha|t|}\right| D^{k} f(t) \mid<\infty\right\},
$$

and similarly define vector-valued versions $\mathbb{E}_{j}^{\alpha}(Z)$.

Recall that $U_{h}:=P_{h} U, W_{h}:=\hat{P}_{h} W$, and $U_{h}=W_{h}$ (the difference between $U$ and $W$, $r(t) \zeta_{0}$, is in the centre space). Further, from (40),

$$
\hat{L}_{\mu, g} U_{h}=L_{\mu, g} U_{h}-\zeta_{1}^{*}\left(U_{h}\right) \zeta_{0}=L_{\mu, g} U_{h} .
$$

So solving

$$
\frac{\partial W_{h}}{\partial t}=\hat{L}_{\mu, g} W_{h}+\hat{P}_{h} F
$$

is equivalent to solving

$$
\frac{\partial U_{h}}{\partial t}=L_{\mu, g} U_{h}+P_{h} F .
$$

Equation (46) has to be solved for $U_{h} \in \mathbb{E}_{0}^{\alpha}\left(\mathbb{D}_{h}\right) \cap \mathbb{E}_{1}^{\alpha}\left(\mathbb{H}_{h}\right)$, for each $\alpha \geq 0$. We know that relevant $F$ have the form $(0, f, 0)^{T}$, where $f \in E_{0}^{\alpha}(\mathbb{R})$, and also have conditions on $U_{h}$ given by the fact that its projections onto the components of the centre space are zero. The calculation is lengthy but follows established arguments [7] and is thus omitted here. We remark that an alternative proof, based on a result by Mielke [12, was given by Calleja and Sire [1].

Lemma 3. Assume $f \in E_{0}^{\alpha}$, for $\alpha \in\left(-\alpha_{0}, \alpha_{0}\right), \alpha_{0}<\delta<p_{0}$; then the system (46) has a unique solution $U_{h} \in E_{0}^{\alpha}\left(\mathbb{D}_{h}\right) \cap E_{1}^{\alpha}\left(\mathbb{H}_{h}\right)$, and the linear map $f \mapsto U_{h}$ from $E_{0}^{\alpha}(\mathbb{R})$ to $E_{0}^{\alpha}\left(\mathbb{D}_{h}\right) \cap E_{1}^{\alpha}\left(\mathbb{H}_{h}\right)$ is bounded uniformly in $\alpha \in\left(-\alpha_{0}, \alpha_{0}\right)$.

3.5. Deriving the normal form. As in Lombardi's book [9, Chapter 8], we use a direct normalisation on (41), thus retaining the benefits of the analyticity of $f$.

We further decompose $W$ before applying the normal form theorem. Let $W=\tilde{c} \zeta_{3}+\tilde{W}$ where $\zeta_{3}^{*}(\tilde{W})=0$. From (29), we can write

$$
\tilde{c}=C+\text { h.o.t. . }
$$

Following Iooss and James [6], we use the coordinate $C$ rather than $\tilde{c}$, where $C$ is a constant of integration which is now treated as a parameter of the system.

We study

$$
\begin{aligned}
Y_{c} & =A \zeta_{1}+B \zeta_{2}+C \zeta_{3}+D \zeta_{q}+\bar{D} \zeta_{-q} \\
& =A \zeta_{1}+B \zeta_{2}+C \zeta_{3}+D_{1} \frac{1}{2}\left(\zeta_{q}+\zeta_{-q}\right)+D_{2} \frac{1}{2 i}\left(\zeta_{q}-\zeta_{-q}\right)
\end{aligned}
$$

Notice that

$$
\begin{aligned}
S((A, B, C, D, \bar{D})) & =(A,-B,-\bar{D},-D), \\
S\left(\left(A, B, C, D_{1}, D_{2}\right)\right) & =\left(A,-B,-D_{1}, D_{2}\right),
\end{aligned}
$$


and that, for the $A, B, C, D_{1}, D_{2}$ coordinates,

$$
\hat{L}_{c}^{0}=\left(\begin{array}{ccccc}
0 & 1 & 0 & 0 & 0 \\
0 & 0 & 1 & 0 & 0 \\
0 & 0 & 0 & 0 & 0 \\
0 & 0 & 0 & 0 & -q \\
0 & 0 & 0 & q & 0
\end{array}\right) .
$$

Theorem 2. A feasible normal form for these coordinates is

$$
\begin{aligned}
\frac{d A}{d t} & =B \\
\frac{d B}{d t} & =C+\phi_{2}\left(A, D_{1}^{2}+D_{2}^{2}, C, \psi\right)+\text { h.o.t. } \\
\frac{d C}{d t} & =0 \\
\frac{d D_{1}}{d t} & =-q D_{2}-q D_{2} \phi_{4}\left(A, D_{1}^{2}+D_{2}^{2}, C, \psi\right)+\text { h.o.t. } \\
\frac{d D_{2}}{d t} & =q D_{1}+q D_{1} \phi_{4}\left(A, D_{1}^{2}+D_{2}^{2}, C, \psi\right)+\text { h.o.t. }
\end{aligned}
$$

where $\phi_{2}$ and $\phi_{4}$ are real polynomials.

The proof uses standard arguments; see, for example, the book by Haragus and Iooss [4.

We now turn to calculating the coefficients in the normal form. Let

$$
\phi_{2}\left(A, D_{1}^{2}+D_{2}^{2}, C, \psi\right)=v A+a A^{2}+c C A+d\left(D_{1}^{2}+D_{2}^{2}\right)+\ldots,
$$

where $v, a, c$ and $d$, etc. are functions of $\psi$. Then the linear operator for the system (48) is

$$
\left(\begin{array}{ccccc}
0 & 1 & 0 & 0 & 0 \\
v & 0 & 1 & 0 & 0 \\
0 & 0 & 0 & 0 & 0 \\
0 & 0 & 0 & 0 & -q \\
0 & 0 & 0 & q & 0
\end{array}\right) .
$$

The eigenvalues are $\pm i q, 0$ and $\pm \sqrt{v}$. Examining the dispersion relation $N(\lambda ; \mu, g)=0$ for $\mu$ close to $1 /(1+4 g)$ (i.e., $v$ close to zero) and looking for roots, $\lambda$, close to zero leads to

$0=\lambda^{2}-2 \mu(\cosh \lambda-1)-2 g \mu(\cosh 2 \lambda-1)=\lambda^{2}(1-\mu(1+4 g))-\lambda^{4} \mu\left(\frac{1}{12}+\frac{16 g}{12}\right)+\ldots$. So

$$
\begin{aligned}
v & =\lambda^{2}=\frac{12(1-\mu(1+4 g))}{(1+16 g) \mu}+\ldots \\
& =\frac{12(1+4 g)(1-\mu(1+4 g))}{(1+16 g)(1-(1-\mu(1+4 g))}+\ldots \\
& =\frac{12(1+4 g)(1-\mu(1+4 g)))}{(1+16 g)}+O\left[(1-\mu(1+4 g))^{2}\right] .
\end{aligned}
$$


Notice that, since we have chosen $-1 / 4<g<-1 / 16$, this expression is negative for $\mu<1 /(1+4 g)$ (supersonic speeds) and positive for $\mu>1 /(1+4 g)$ (subsonic speeds). This is the reverse of the case in Iooss' paper 7 .

We now calculate the coefficient $a$ in the normal form. Let $\hat{M}_{2}(W, W)$ represent the quadratic part of $G_{1}^{\prime}\left(\delta^{1} X-x\right)-G_{1}^{\prime}\left(x-\delta^{-1} X\right)+g\left[G_{2}^{\prime}\left(\delta^{2} X-x\right)-G_{2}^{\prime}\left(x-\delta^{-2} X\right)\right]$. In summary, the relevant parts of the normal form, etc. we are looking at are

$$
\begin{aligned}
\frac{d A}{d t} & =B \\
\frac{d B}{d t} & =C+v A+a A^{2}+\ldots \\
\frac{d C}{d t} & =0 \\
\frac{d D_{1}}{d t} & =-q D_{2}-q D_{2} \phi_{4}\left(A, D_{1}^{2}+D_{2}^{2}, C\right)+\ldots \\
\frac{d D_{2}}{d t} & =q D_{1}+q D_{1} \phi_{4}\left(A, D_{1}^{2}+D_{2}^{2}, C\right)+\ldots .
\end{aligned}
$$

The differential equation is

$$
\frac{d W}{d t}=\hat{L}^{0} W+\hat{M}_{2}(W, W)+\ldots
$$

where

$$
\begin{aligned}
W= & Y_{c}+Y_{h}+\Phi\left(Y_{c}, \psi\right) \\
= & A \zeta_{1}+B \zeta_{2}+C \zeta_{3}+D_{1} \frac{1}{2}\left(\zeta_{q}+\zeta_{-q}\right)+D_{2} \frac{1}{2 i}\left(\zeta_{q}-\zeta_{-q}\right) \\
& +A^{2} \Phi_{20000}+A B \Phi_{11000}+A C \Phi_{10100}+\cdots+Y_{h}
\end{aligned}
$$

We now identify coefficients of $A^{2}$ and $A B$ in the differential equation to give

$$
\begin{aligned}
a \zeta_{2} & =\hat{L}_{c}^{0} \Phi_{20000}+\hat{M}_{2}\left(\zeta_{1}, \zeta_{1}\right) \\
2 \Phi_{20000} & =\hat{L}_{c}^{0} \Phi_{11000}+2 \hat{M}_{2}\left(\zeta_{1}, \zeta_{2}\right) .
\end{aligned}
$$

We notice that, for $\zeta_{1},\left(\delta^{1} X-x\right)^{2}-\left(x-\delta^{-1} X\right)^{2}$ and $\left(\delta^{2} X-x\right)^{2}-\left(x-\delta^{-2} X\right)^{2}$ are both zero, so $\hat{M}_{2}\left(\zeta_{1}, \zeta_{1}\right)=0$ and (49) becomes

$$
a \zeta_{2}=\hat{L}_{c}^{0} \Phi_{20000}
$$

We also need the kernel of $\left(\hat{L}_{c}^{0}\right)^{*}$,

$$
\left(\hat{L}_{c}^{0}\right)^{*}=\left(\begin{array}{ccccc}
0 & 0 & 0 & 0 & 0 \\
1 & 0 & 0 & 0 & 0 \\
0 & 1 & 0 & 0 & 0 \\
0 & 0 & 0 & 0 & q \\
0 & 0 & 0 & -q & 0
\end{array}\right) .
$$

The kernel of $\left(\hat{L}_{c}^{0}\right)^{*}$ in the $\zeta$ basis is thus spanned by $(0,0,1,0,0)^{T}$, i.e., by $\zeta_{3}$.

This gives that, for any $W$,

$$
\left(\hat{L}_{c}^{0} W, \zeta_{3}\right)=\left(W,\left(\hat{L}_{c}^{0}\right)^{*} \zeta_{3}\right)=0 .
$$


Inverting (51) gives

$$
\Phi_{20000}=a \zeta_{3}+\tilde{a} \zeta_{1},
$$

where $\tilde{a}$ is a constant.

Substituting in the equation (50) for the coefficients of $A B$ gives

$$
2 a \zeta_{3}+\tilde{a} \zeta_{1}=\hat{L}_{c}^{0} \Phi_{11000}+2 \hat{M}_{2}\left(\zeta_{1}, \zeta_{2}\right)
$$

and if we now apply $\zeta_{3}^{*}$, we get

$$
2 a=\zeta_{3}^{*}\left(2 \hat{M}_{2}\left(\zeta_{1}, \zeta_{2}\right)\right)
$$

We now need to calculate $\zeta_{3}^{*}\left(\hat{M}_{2}\left(\zeta_{1}, \zeta_{2}\right)\right)$. Let $\zeta_{j}=\left(x_{j}, \xi_{j}, X_{j}\right)$; then

$$
\begin{array}{r}
\left(\delta^{1} X_{1}-x_{1}\right)\left(\delta^{1} X_{2}-x_{2}\right)-\left(x_{1}-\delta^{-1} X_{1}\right)\left(x_{2}-\delta^{-1} X_{2}\right)=1 \cdot \frac{1}{2}-(-1) \cdot \frac{1}{2}=1, \\
\left(\delta^{2} X_{1}-x_{1}\right)\left(\delta^{2} X_{2}-x_{2}\right)-\left(x_{1}-\delta^{-2} X_{1}\right)\left(x_{2}-\delta^{-2} X_{2}\right)=8 .
\end{array}
$$

Hence

$$
\hat{M}_{2}\left(\zeta_{1}, \zeta_{2}\right)=\left(0,\left(\alpha_{1}+8 g \alpha_{2}\right), 0\right)^{T}
$$

and, using the formula derived for $\xi_{3}^{*}$,

$$
a=\frac{-12(1+4 g)}{(1+16 g)}\left(\alpha_{1}+8 g \alpha_{2}\right) .
$$

If we assume that (7) holds, then $\alpha_{1}+8 g \alpha_{2}>0$, and thus $a>0$. We are now in a position to take the special case $C=0$ and then apply the scaling used in Lombardi's book [9, page 322] for $\psi>0$ and prove the persistence of the subsonic solitary solutions homoclinic to exponentially small periodic solutions.

Lombardi's proof needs one more adaption for our non-sectorial spectrum; i.e., we need to show that the equation (8.35) in [9] has a unique $2 \pi$-periodic solution in the hyperbolic space for any $F$ of the form $(0, f, 0)^{T}$ as before. In our notation, this equation is

$$
\frac{q_{0, \sqrt{v}}}{\sqrt{v}} \frac{\partial Z_{h}}{\partial s}-\frac{\hat{L}_{h}^{0}}{\sqrt{v}} Z_{h}=F_{h},
$$

where $q_{0, \sqrt{v}}=q+O(\sqrt{v})$. (The constants $q_{0, \sqrt{v}}, \sqrt{v}$, which are dependent on the parameter $\psi$, and the variable $s$ are the result of a scaling used in [9] to simplify the normal form in the centre space and to ensure that we are working with functions of periodicity $2 \pi$.)

The working to find the form of $Z_{h}=\left(x_{h}, \xi_{h}, X_{h}\right)^{T}$ is essentially the same as before, just adjusted for the constants in the equation due to the new scaling and using Fourier series rather than Fourier transforms.

Examining the identity for $\hat{\xi}_{h, n}$ (the $n$th Fourier coefficient for $\xi_{h}$ ), we find

$$
\frac{\hat{x}_{h, n}}{\sqrt{v}}\left(n^{2} q_{0, \sqrt{v}}^{2}-2 \mu(1+g)+2 \mu \cos \left(n q_{0, \sqrt{v}}\right)+2 \mu g \cos \left(2 n q_{0, \sqrt{v}}\right)\right) \leq\left(\frac{C_{1}(q)}{n}+C_{2}(q)\right)\left|\hat{f}_{n}\right| \text {. }
$$

The coefficient of $\hat{x}_{h, n}$ here is $N\left(i n q_{0, \sqrt{v}}, \mu, g\right)$, which is $O\left(n^{2}\right)$ as $n \rightarrow \infty$, and nonzero if $n \neq 0$, or $n \neq \pm 1$ and $v \neq 0$. If the opposite to either of these statements holds, then we 
would have contributions in the Fourier expansion for $Z_{h}$ from the centre space, so their coefficients would have to be zero. Otherwise, we have

$$
\hat{x}_{h, n} \leq \frac{C(q) \sqrt{v}}{n^{3}}\left|\hat{f}_{n}\right|
$$

and the proof can proceed as in [9, Chapter 8], leading to a proof of Theorem 1 .

4. Existence of supersonic periodic solutions. We now study (9) for $\mu$ close to and strictly less than $1 /(1+4 g)$, again for fixed $g \in\left(-\frac{1}{4}+\frac{1}{4 \pi^{2}},-\frac{1}{16}\right)$. The travelling wave is then supersonic in the terminology introduced after Theorem In this section, we show the following result.

Theorem 3. Suppose the potentials satisfy (4) -(7). Also note that the nonlinearity $M_{\mu, g}$, as in (10), is in $C^{k}(\mathbb{D}, \mathbb{D})$ for $k \geq 1$ and $\left\|M_{\mu, g} U\right\|_{\mathbb{D}} \leq c(\rho)\|U\|_{\mathbb{D}}^{2}$ for all $U \in \mathbb{D}$ with $\|u\|_{\mathbb{D}} \leq \rho$, where $\rho$ is an arbitrary constant. (In our case, the latter condition is ensured by $G_{1}$ and $G_{2} \in C^{2}(I)$, where $I$ denotes an open neighbourhood of $0 \in \mathbb{R}$.) For $g$ such that $-\frac{1}{4}+\frac{1}{4 \pi^{2}}<g<-\frac{1}{16}$ there exists an $\eta_{g}>0$ depending on $g$ and $c_{0}$ such that, if $c_{0}^{2}(1+4 g)<c^{2}<c_{0}^{2}(1+4 g)+\eta_{g}$, there exists at least one one-parameter family of smooth periodic waves as solutions to (3).

Notice that the conditions on the potentials have been considerably relaxed compared with Theorem 1 and also that the condition on $c$ is equivalent to $\mu_{g}<\mu<1 /(1+4 g)$.

A similar result has recently been proved with a linking argument [15] where an estimate is made for $\eta_{g}$, so we only sketch the argument using centre manifold techniques.

To show the existence of supersonic periodic solutions, we no longer need to treat $\mu$ as a small parameter, but just choose any value for which the linear equation has supersonic periodic solutions and the centre space is spanned by the six vectors $\zeta_{0}$ and $\zeta_{1}$ from (27) and

$$
\zeta_{ \pm q_{1}}=\left(\begin{array}{c}
1 \\
\pm i q_{1} \\
e^{ \pm i q_{1}}
\end{array}\right), \quad \zeta_{ \pm q_{2}}=\left(\begin{array}{c}
1 \\
\pm i q_{2} \\
e^{ \pm i q_{2}}
\end{array}\right),
$$

where $0<q_{1}<q_{2}$. We need the projection $P$ onto the centre space, and we can think of it as $P=P_{0,2}+P_{1}+P_{2}$, where $P_{0,2}$ is the projection onto the subspace spanned by $\zeta_{0}$ and $\zeta_{1}$ and $P_{1}$ is the projection onto the subspace spanned by $\zeta_{ \pm q_{1}}$, etc. So we seek $P F$ where $F=\left(f_{0}, f_{1}, F_{2}\right)^{T}$. These formulae are found using the same methods as before.

We again use the invariance under the shift operator (36) to decompose $U$ as in (37). We look for solutions in $\hat{\mathbb{H}}$, the co-dimension-one subspace of $\mathbb{H}$ containing $W$. The component along $\zeta_{0}$ of the full solution represents a uniform stretching or contraction of the lattice.

Recall that (9) becomes the system (38)-(39), with $\hat{L}_{\mu, g}$ as in (40); $\hat{L}_{\mu, g}$ has the same spectrum as $L_{\mu, g}$ except that, for this choice of $\mu$, the eigenvalue at 0 is now single rather than double and

$$
\hat{L}_{\mu, g} \zeta_{1}=0 \text {. }
$$

This time, we wish to use the Iooss and Vanderbauwhede Theorem [14] to apply a centre manifold reduction so that we are working in a finite-dimensional space. To use 
this theorem, we need to separate (9) into a central and a hyperbolic part and show that the hyperbolic part has a unique solution in the relevant spaces. The method of proof is exactly the same as before and ensures that the centre manifold reduction is feasible.

We are now working in a five-dimensional space. We again follow Iooss and James [6] and further decompose $W$ before applying the normal form. In this centre space, the projection onto $\zeta_{1}$ enables us (analogously to the homoclinic case) to decompose $W$ into $\tilde{a} \zeta_{1}+\tilde{W}$, where

$$
\tilde{a}=A+\text { h.o.t. , }
$$

and $A$ is a constant of integration. We use $A$ as the coordinate along $\zeta_{1}$ for the normal form and treat it as a parameter for the system. We can then look at the case $A=0$. We are effectively looking at a four-dimensional centre space with eigenvalues $\pm i q_{1}$ and $\pm i q_{2}$, and we can use the Devaney-Lyapunov Theorem (e.g., [9, Theorem 4.1.1]). Specifically, the eigenvalue $i q_{2}$ satisfies the conditions of the Devaney-Lyapunov Theorem and gives us one family of periodic solutions in $\hat{\mathbb{H}}$. If $q_{2} / q_{1}$ is non-integer (a dense set), the eigenvalue $i q_{1}$ gives us a second family of periodic solutions. For the case $r(t)=0$ these solutions are also periodic in $\mathbb{H}$. This proves Theorem 3 ,

\section{REFERENCES}

[1] Renato Calleja and Yannick Sire, Travelling waves in discrete nonlinear systems with nonnearest neighbour interactions, Nonlinearity 22 (2009), no. 11, 2583-2605, DOI 10.1088/09517715/22/11/001. MR2550686 (2010j:82069)

[2] N. Flytzanis, S. Pnevmatikos, and M. Remoissenet. Kink, breather and asymmetric envelope or dark solitons in nonlinear chains. I. Monatomic chain. J. Phys. C Solid State, 18(24):4603, 1985.

[3] G. Gaeta, C. Reiss, M. Peyrard, and T. Dauxois. Simple models of non-linear DNA dynamics. La Rivista del Nuovo Cimento (1978-1999), 17(4):1-48, 1994.

[4] Mariana Haragus and Gérard Iooss, Local bifurcations, center manifolds, and normal forms in infinite-dimensional dynamical systems, Universitext, Springer-Verlag London Ltd., London, 2011. MR.2759609 (2012d:37193)

[5] Gérard Iooss, Travelling waves in the Fermi-Pasta-Ulam lattice, Nonlinearity 13 (2000), no. 3, 849-866, DOI 10.1088/0951-7715/13/3/319. MR.1759004 (2002a:37114)

[6] Gérard Iooss and Guillaume James, Localized waves in nonlinear oscillator chains, Chaos 15 (2005), no. 1, 015113, 15, DOI 10.1063/1.1836151. MR2133464 (2005m:37179)

[7] Gérard Iooss and Klaus Kirchgässner, Travelling waves in a chain of coupled nonlinear oscillators, Comm. Math. Phys. 211 (2000), no. 2, 439-464, DOI 10.1007/s002200050821. MR.1754524 (2002b:37124)

[8] Tosio Kato, Perturbation theory for linear operators, Classics in Mathematics, Springer-Verlag, Berlin, 1995. Reprint of the 1980 edition. MR 1335452 (96a:47025)

[9] Eric Lombardi, Oscillatory integrals and phenomena beyond all algebraic orders. With applications to homoclinic orbits in reversible systems, Lecture Notes in Mathematics, vol. 1741, Springer-Verlag, Berlin, 2000. MR 1770093 (2002f:34081)

[10] Percy D. Makita, Periodic and homoclinic travelling waves in infinite lattices, Nonlinear Anal. 74 (2011), no. 6, 2071-2086, DOI 10.1016/j.na.2010.11.011. MR.2781738(2012c:37160)

[11] Percy Makita. Subharmonics and homoclinics for a class of Hamiltonian-like equations. Technical Report 37/2009, Max-Planck Institut für Mathematik in den Naturwissenschaften, Leipzig, 2009.

[12] Alexander Mielke, Über maximale $L^{p}$-Regularität für Differentialgleichungen in Banach- und Hilbert-Räumen, Math. Ann. 277 (1987), no. 1, 121-133, DOI 10.1007/BF01457282 (German). MR884650(88i:34117)

[13] E. K. H. Salje. Multi-scaling and mesoscopic structures. Philosophical Transactions of the Royal Society A: Mathematical, Physical and Engineering Sciences, 368(1914):1163-1174, 2010. 
[14] A. Vanderbauwhede and G. Iooss, Center manifold theory in infinite dimensions, Dynamics reported: expositions in dynamical systems, Dynam. Report. Expositions Dynam. Systems (N.S.), vol. 1, Springer, Berlin, 1992, pp. 125-163. MR1153030 (93f:58174)

[15] Christine R. Venney and Johannes Zimmer, Persistence of supersonic periodic solutions for chains with anharmonic interaction potentials between neighbours and next to nearest neighbours, Dyn. Syst. 26 (2011), no. 4, 503-518. MR.2852938

[16] Jonathan A. D. Wattis, Approximations to solitary waves on lattices. III. The monatomic lattice with second-neighbour interactions, J. Phys. A 29 (1996), no. 24, 8139-8157, DOI 10.1088/03054470/29/24/035. MR.1446913 (98c:82048) 